\title{
Nonoscillatory solutions for first-order neutral dynamic equations with continuously distributed delay on time scales
}

\section{Zhanhe Chen ${ }^{1}$, Jingjiang LV ${ }^{1}$, Xuanli He ${ }^{1 *}$ (D) and Ting Li ${ }^{1}$}

"Correspondence:

hxlczh@gxu.edu.cn

${ }^{1}$ College of Mathematics and

Information Science, Guangx

University, Nanning, P.R. China

\section{Abstract}

In this paper, we establish the existence of nonoscillatory solutions to the neutral dynamic equation

$$
\left[x(t)-\int_{a}^{b} p(t, \eta) x(g(t, \eta)) \Delta \eta\right]^{\Delta}+\int_{c}^{d} \omega(t, v) x(h(t, v)) \Delta v=0
$$

on a time scale $\mathbb{T}$. Some examples are given to illustrate the main results.

MSC: 34K11;39A10; 39A99

Keywords: Neutral dynamic equation; Nonoscillatory solution; Time scale

\section{Introduction}

In this paper, we will consider the existence of nonoscillatory solutions to the first-order neutral dynamic equation of the form

$$
\left[x(t)-\int_{a}^{b} p(t, \eta) x(g(t, \eta)) \Delta \eta\right]^{\Delta}+\int_{c}^{d} \omega(t, v) x(h(t, v)) \Delta v=0
$$

on a time scale $\mathbb{T}$, where $a, b, c, d \in \mathbb{T}$ with $a \leq b$ and $c \leq d$ and $p(t, \eta), g(t, \eta), \omega(t, \eta), h(t, \eta) \in$ $C(\mathbb{T}, \mathbb{T})$. Throughout this paper, we assume that $\mathbb{T}=\left[t_{0}, \infty\right)_{\mathbb{T}}:=\left\{t \in \mathbb{T}: t \geq t_{0}\right\}$.

In recent years, there has been much research activity concerning the nonoscillation of solutions of various equations on time scales, and we refer the reader to [1-4]. Mathsen, Wang and Wu [5] established some sufficient conditions for the existence of positive solutions of the delay equation

$$
[x(t)+p(t) x(g(t))]^{\Delta}+q(t) x(h(t))=0 .
$$

Zhu and Wang [6] established the existence of nonoscillatory solutions to the neutral equation

$$
[x(t)+p(t) x(g(t))]^{\Delta}+f(t, x(h(t)))=0 .
$$

(c) The Author(s) 2019. This article is distributed under the terms of the Creative Commons Attribution 4.0 International License (http://creativecommons.org/licenses/by/4.0/), which permits unrestricted use, distribution, and reproduction in any medium, provided you give appropriate credit to the original author(s) and the source, provide a link to the Creative Commons license, and indicate if changes were made. 
Gao, Chen and Shi [7] established the existence of oscillatory solutions to the neutral equation

$$
\left[r(t)\left[x(t)+\int_{a}^{b} p(t, \mu) x[\tau(t, \mu)] d \mu\right]^{\prime \prime}\right]^{\prime}+\int_{c}^{d} q(t, \xi) f(x[\sigma(t, \xi)]) d \xi=0 .
$$

Up to now there have been few studies on equations with continuously distributed delay on time scales. For some related works, the reader is referred to the papers [8-10]. In this paper, we obtain some new sufficient conditions for the existence of nonoscillatory solutions of equation (1.1). The method used in this paper is motivated by the work of Zhu and Wang in [6]. And our work enriches the research with the case of equations with continuously distributed delay on time scales.

This paper is organized as follows. In Sect. 2, we recall some basic concepts and some preliminaries briefly. In Sect. 3, we focus on the study of sufficient conditions for the existence of nonoscillatory solutions to the first-order neutral dynamic equation (1.1). In Sect. 4, some examples are presented to illustrate our main results for equation (1.1).

\section{Preliminaries}

For convenience, we recall some concepts related to time scales. More details can be found in $[11,12]$.

Definition 2.1 (see [12, Definition 1.10]) Assume $f: \mathbb{T} \rightarrow \mathbb{R}$ is a function and $t \in \mathbb{T}^{\kappa}$. Then we define $f^{\Delta}(t)$ to be the number (provided it exists) with the property that given any $\varepsilon>0$, there is a neighborhood $U$ of $\mathrm{t}$ (i.e., $U=(t-\delta, t+\delta) \cap \mathbb{T}$ for some $\delta>0)$ such that

$$
\left|[f(\sigma(t))-f(s)]-f^{\Delta}(t)[\sigma(t)-s]\right| \leq \varepsilon|\sigma(t)-s|, \quad \text { for all } s \in U \text {. }
$$

We call $f^{\Delta}(t)$ the delta (or Hilger) derivative of $f$ at $t$. Moreover, we say that $f$ is delta (or Hilger) differentiable (or, in short, differentiable) on $\mathbb{T}^{\kappa}$ provided $f^{\Delta}(t)$ exists for all $t \in \mathbb{T}^{\kappa}$. The function $f^{\Delta}: \mathbb{T}^{\kappa} \rightarrow \mathbb{R}$ is then called the (delta) derivative of $f$ on $\mathbb{T}^{\kappa}$.

Using the definition of delta derivative, it's easy to see the following results (see [12, Theorem 1.16]). If $f$ is continuous at $t \in \mathbb{T}$ and $t$ is right-scattered, then $f$ is differentiable at $t$ with

$$
f^{\Delta}(t)=\frac{f(\sigma(t))-f(t)}{\mu(t)} .
$$

Moreover, if $t$ is right-dense then $f$ is differentiable at $t$ iff the limit

$$
\lim _{s \rightarrow t} \frac{f(t)-f(s)}{t-s}
$$

exists as a finite number. In this case

$$
f^{\Delta}(t)=\lim _{s \rightarrow t} \frac{f(t)-f(s)}{t-s} .
$$


In the sequel, as in [6], we denote by $C\left(\left[t_{0}, \infty\right)_{\mathbb{T}}, \mathbb{R}\right)$ all continuous functions mapping $\left[t_{0}, \infty\right)_{\mathbb{T}}$ into $\mathbb{R}$, and let

$$
B C\left[t_{0}, \infty\right)_{\mathbb{T}}=\left\{x \in C\left(\left[t_{0}, \infty\right)_{\mathbb{T}}, \mathbb{R}\right): \sup _{t \in\left[t_{0}, \infty\right)_{\mathbb{T}}}|x(t)|<\infty\right\} .
$$

Endowing $B C\left[t_{0}, \infty\right)_{\mathbb{T}}$ with the norm $\|x\|=\sup _{t \in\left[t_{0}, \infty\right)_{\mathbb{T}}}|x(t)|$ makes $\left(B C\left[t_{0}, \infty\right)_{\mathbb{T}},\|\cdot\|\right)$ a Banach space. To prove our main results, we need the following lemmas.

Lemma 2.1 (see [6, Lemma 4]) Suppose that $X \subseteq B C\left[t_{0}, \infty\right)_{\mathbb{T}}$ is bounded and uniformly Cauchy. Further, suppose that $X$ is equi-continuous on $\left[t_{0}, t_{1}\right]_{\mathbb{T}}$ for any $t_{1} \in\left[t_{0}, \infty\right)_{\mathbb{T}}$. Then $X$ is relatively compact.

Lemma 2.2 (see [13, Krasnoselskii's Fixed Point Theorem]) Suppose that $\Omega$ is a Banach space and $X$ is a bounded, convex and closed subset of $\Omega$. Suppose further that there exist two operators $U, S: X \rightarrow \Omega$ such that

(i) $U x+$ Sy $\in X$ for all $x, y \in X$;

(ii) $U$ is a contraction mapping;

(iii) $S$ is completely continuous.

Then $U+S$ has a fixed point in $X$.

\section{Main results}

Theorem 3.1 Assume that $p(t, \eta)>0, \omega(s, v)>0$ for any $t \in \mathbb{T}, \eta \in[a, b]_{\mathbb{T}}$ and $v \in[c, d]_{\mathbb{T}}$. And suppose further that there exists a constant $\alpha$ such that

$$
\int_{a}^{b} p(t, \eta) \Delta \eta \leq \alpha<1
$$

and

$$
\int_{t_{0}}^{\infty} \int_{c}^{d} \omega(s, v) \Delta v \Delta s \leq \frac{1-\alpha}{2} .
$$

Then (1.1) has a bounded nonoscillatory solution.

Proof We define the Banach space $B C\left[t_{0}, \infty\right)_{\mathbb{T}}$ as in $(2.1)$ and let

$$
X=\left\{x \in B C\left[t_{0}, \infty\right)_{\mathbb{T}}: \frac{1-\alpha}{2} \leq x(t) \leq 1\right\} .
$$

It is easy to check that $X$ is a bounded, convex and closed subset of $B C\left[t_{0}, \infty\right)_{\mathbb{T}}$. We will apply the Krasnoselskii's Fixed Point Theorem to obtain the desired result. To this end, first of all, we define two operators $U, S: X \rightarrow B C\left[t_{0}, \infty\right)_{\mathbb{T}}$ as follows:

$$
(U x)(t)=\int_{a}^{b} p(t, \eta) x(g(t, \eta)) \Delta \eta
$$

and

$$
(S x)(t)=\frac{1-\alpha}{2}+\int_{t}^{\infty} \int_{c}^{d} \omega(s, v) x(h(s, v)) \Delta v \Delta s .
$$

And then, we will check that $U$ and $S$ satisfy the conditions in Lemma 2.2. 
(1) For $x, y \in X$, from (3.1) and (3.2), we have

$$
\frac{1-\alpha}{2} \leq(U x)(t)+(S y)(t) \leq \alpha+\frac{1-\alpha}{2}+\frac{1-\alpha}{2}=1, \quad \text { for any } t \in \mathbb{T} .
$$

So $U x+S y \in X$ for any $x, y \in X$.

(2) For $x, y \in X$ and $t \in \mathbb{T}$, we have

$$
|(U x)(t)-(U y)(t)| \leq\left|\int_{a}^{b} p(t, \eta) \Delta \eta\right|\|x-y\| \leq \alpha\|x-y\|
$$

which implies that $U$ is a contraction mapping on $X$.

(3) We now show that $S$ is completely continuous. Clearly, $S$ maps $X$ into itself.

Let $x_{n}, x \in X$ and $\left\|x_{n}-x\right\| \rightarrow 0$ as $n \rightarrow \infty$. Then, from (3.2), we get that

$$
\begin{aligned}
\left|\left(S x_{n}\right)(t)-(S x)(t)\right| & \leq \int_{t}^{\infty} \int_{c}^{d} \omega(s, v)\left|x_{n}(h(s, v))-x(h(s, v))\right| \Delta v \Delta s \\
& \leq \frac{1-\alpha}{2}\left\|x_{n}-x\right\|,
\end{aligned}
$$

which implies that $S$ is continuous on $X$.

For any $\varepsilon>0$, by (3.2), we can choice $t_{1} \in\left[t_{0}, \infty\right)_{\mathbb{T}}$ such that

$$
\int_{t_{1}}^{\infty} \int_{c}^{d} \omega(s, v) \Delta v \Delta s<\frac{\varepsilon}{2}
$$

Then, for any $x \in X$ and $u, v \in\left[t_{1}, \infty\right)_{\mathbb{T}}$, we have

$$
\begin{aligned}
|(S x)(u)-(S x)(v)| \leq & \left|\int_{u}^{\infty} \int_{c}^{d} \omega(s, v) x(h(s, v)) \Delta v \Delta s\right| \\
& +\left|\int_{v}^{\infty} \int_{c}^{d} \omega(s, v) x(h(s, v)) \Delta v \Delta s\right| \\
& \leq\left|\int_{u}^{\infty} \int_{c}^{d} \omega(s, v) \Delta v \Delta s\right|+\left|\int_{v}^{\infty} \int_{c}^{d} \omega(s, v) \Delta v \Delta s\right| \\
& <\varepsilon .
\end{aligned}
$$

Hence, $S X$ is uniformly Cauchy.

For any $t_{2} \in\left[t_{0}, \infty\right)_{\mathbb{T}}$, by (3.2), for any $s \in\left[t_{0}, \infty\right)_{\mathbb{T}}$, we have that $\int_{c}^{d} \omega(s, v) \Delta v \leq N$ for some positive constant $N$. And so, for any $\varepsilon>0$, take $\delta=\varepsilon / N$, then for any $x \in X$, when $u, v \in\left[t_{0}, t_{2}\right]$ with $|u-v|<\delta$, we have

$$
|(S x)(u)-(S x)(v)|=\left|\int_{u}^{v} \int_{c}^{d} \omega(s, v) x(h(s, v)) \Delta v \Delta s\right| \leq N|v-u|<\varepsilon .
$$

Thus $S X$ is equi-continuous on $\left[t_{0}, t_{2}\right]_{\mathbb{T}}$.

By Lemma 2.1, $S X$ is relatively compact. And so $S$ is a completely continuous mapping. Finally, by Lemma 2.2 , there exists $x \in X$ such that $(U+S) x=x$, which implies that $x(t)$ is a bounded nonoccillatory solution of (1.1). The proof is complete. 
Theorem 3.2 Assume that $p(t, \eta)<0, \omega(s, v)>0$ for any $t \in \mathbb{T}, \eta \in[a, b]_{\mathbb{T}}$ and $v \in[c, d]_{\mathbb{T}}$. And suppose further that there exists a constant $\alpha$ such that (3.2) and

$$
-1<-\alpha \leq \int_{a}^{b} p(t, \eta) \Delta \eta
$$

hold. Then (1.1) has a bounded nonoscillatory solution.

Proof We define the Banach space $B C\left[t_{0}, \infty\right)_{\mathbb{T}}$ as in $(2.1)$ and let

$$
X=\left\{x \in B C\left[t_{0}, \infty\right)_{\mathbb{T}}: \frac{1-\alpha}{2} \leq x(t) \leq 1\right\} .
$$

It is easy to check that $X$ is a bounded, convex and closed subset of $B C\left[t_{0}, \infty\right)_{\mathbb{T}}$.

Now we define two operators $U$ and $S: X \rightarrow B C\left[t_{0}, \infty\right)_{\mathbb{T}}$ as follows:

$$
(U x)(t)=\int_{a}^{b} p(t, \eta) x(g(t, \eta)) \Delta \eta
$$

and

$$
(S x)(t)=\frac{1+\alpha}{2}+\int_{t}^{\infty} \int_{c}^{d} \omega(s, v) x(h(s, v)) \Delta v \Delta s .
$$

Similar to the proof of Theorem 3.1, we can show that $U$ and $S$ satisfy the conditions in Lemma 2.2, which implies the desired result. The proof is complete.

Theorem 3.3 Assume that $p(t, \eta)>0, \omega(s, v)<0$ for any $t \in \mathbb{T}, \eta \in[a, b]_{\mathbb{T}}$ and $v \in[c, d]_{\mathbb{T}}$. And suppose further that there exists a constant $\alpha$ such that (3.1) and

$$
\int_{t_{0}}^{\infty} \int_{c}^{d} \omega(s, v) \Delta v \Delta s \geq \frac{\alpha-1}{4}
$$

hold. Then (1.1) has a bounded nonoscillatory solution.

Proof We define the Banach space $B C\left[t_{0}, \infty\right)_{\mathbb{T}}$ as in $(2.1)$ and let

$$
X=\left\{x \in B C\left[t_{0}, \infty\right)_{\mathbb{T}}: \frac{1-\alpha}{4} \leq x(t) \leq 1\right\} .
$$

It is easy to check that $X$ is a bounded, convex and closed subset of $B C\left[t_{0}, \infty\right)_{\mathbb{T}}$.

Now we define two operators $U$ and $S: X \rightarrow B C\left[t_{0}, \infty\right)_{\mathbb{T}}$ as follows:

$$
(U x)(t)=\int_{a}^{b} p(t, \eta) x(g(t, \eta)) \Delta \eta
$$

and

$$
(S x)(t)=\frac{1-\alpha}{2}+\int_{t}^{\infty} \int_{c}^{d} \omega(s, v) x(h(s, v)) \Delta v \Delta s .
$$


Similar to the proof of Theorem 3.1, we can show that $U$ and $S$ satisfy the conditions in Lemma 2.2, which implies the desired result. The proof is complete.

Theorem 3.4 Assume that $p(t, \eta)<0, \omega(s, v)<0$ for any $t \in \mathbb{T}, \eta \in[a, b]_{\mathbb{T}}$ and $v \in[c, d]_{\mathbb{T}}$. And suppose further that there exists a constant $\alpha$ such that (3.3) and (3.4) hold, then (1.1) has a bounded nonoscillatory solution.

Proof We define the Banach space $B C\left[t_{0}, \infty\right)_{\mathbb{T}}$ as in $(2.1)$ and let

$$
X=\left\{x \in B C\left[t_{0}, \infty\right)_{\mathbb{T}}: \frac{1-\alpha}{4} \leq x(t) \leq 1\right\}
$$

It is easy to check that $X$ is a bounded, convex and closed subset of $B C\left[t_{0}, \infty\right)_{\mathbb{T}}$.

Now we define two operators $U$ and $S: X \rightarrow B C\left[t_{0}, \infty\right)_{\mathbb{T}}$ as follows:

$$
(U x)(t)=\int_{a}^{b} p(t, \eta) x(g(t, \eta)) \Delta \eta
$$

and

$$
(S x)(t)=\frac{1+\alpha}{2}+\int_{t}^{\infty} \int_{c}^{d} \omega(s, v) x(h(s, v)) \Delta v \Delta s
$$

Similar to the proof of Theorem 3.1, we can show that $U$ and $S$ satisfy the conditions in Lemma 2.2, which implies the desired result. The proof is complete.

\section{Examples}

Example 4.1 Let $q>1$ and $\mathbb{T}=q^{\mathbb{N}}$. For some fixed natural number $k$, we consider the following equation:

$$
\left[x(t)-\int_{q}^{q^{k}} \frac{x(t+\eta)}{(t+2 \eta) \eta} \Delta \eta\right]^{\Delta}+\int_{q}^{q^{k}} \frac{x(t+2 v)}{t^{2} v(t+4 v)} \Delta v=0
$$

i.e., $p(t, \eta)=\frac{1}{(t+2 \eta) \eta}, \omega(t, v)=\frac{1}{t^{2} v(t+4 v)}, a=q=c, b=q^{k}=d$. Then we have

$$
\int_{a}^{b} p(t, \eta) \Delta \eta \leq \int_{q}^{q^{k}} \frac{1}{2 \eta^{2}} \Delta \eta=\frac{1}{2}\left(1-\frac{1}{q^{k-1}}\right)<\frac{1}{2}, \quad t \in \mathbb{T},
$$

and

$$
\int_{t_{0}}^{\infty} \int_{c}^{d} \omega(s, v) \Delta v \Delta s<\int_{q}^{\infty} \frac{1}{s^{2}} \Delta s \int_{q}^{q^{k}} \frac{1}{4 v^{2}} \Delta v=\frac{1}{4}\left(1-\frac{1}{q^{k-1}}\right)<\frac{1}{4}
$$

Therefore, by Theorem 3.1 with $\alpha=\frac{1}{2}$, (4.1) has a bounded nonoscillatory solution.

Example 4.2 Let $\mathbb{T}=\mathbb{N}^{2}$. Consider the following equation:

$$
\left[x(t)+\int_{1}^{9} \frac{x(t+\eta-1)}{5(t+2 \eta) \eta} \Delta \eta\right]^{\Delta}+\int_{1}^{9} \frac{3 x\left(t+\frac{1}{2} v-1\right)}{10 t(1+\sqrt{t})^{2}(t+2 v) v} \Delta v=0
$$


i.e., $p(t, \eta)=\frac{-1}{5(t+2 \eta) \eta}, \omega(t, v)=\frac{3}{10 t(1+\sqrt{t})^{2}(t+2 v) v}, a=1=c, b=9=d$. Then we have

$$
\int_{a}^{b} p(t, \eta) \Delta \eta \geq \int_{1}^{9} \frac{-1}{5(t+2 \sqrt{\eta}) \eta} \Delta \eta=-\frac{1}{4}, \quad t \in \mathbb{T}
$$

and

$$
\int_{t_{0}}^{\infty} \int_{c}^{d} \omega(s, v) \Delta v \Delta s \leq \int_{1}^{\infty} \frac{3}{10 s(1+\sqrt{s})^{2}} \Delta s \int_{1}^{9} \frac{1}{(1+2 v) v} \Delta v=\frac{3}{8}
$$

Therefore, by Theorem 3.2 with $\alpha=\frac{1}{4}$, (4.2) has a bounded nonoscillatory solution.

Example 4.3 Let $\mathbb{T}=\mathbb{N}^{\frac{1}{2}}$. Consider the following equation

$$
\left[x(t)-\int_{1}^{\sqrt{3}} \frac{\eta}{t+\eta} x(t+\eta) \Delta \eta\right]^{\Delta}-\int_{1}^{\infty} \int_{1}^{\sqrt{3}} \frac{v x(t+3 v)}{4 t \sqrt{1+t^{2}}(t+v)} \Delta v=0
$$

i.e., $p(t, \eta)=\frac{\eta}{t+\eta}, \omega(t, v)=-\frac{v}{4 t \sqrt{1+t^{2}}(t+v)}, a=1=c, b=\sqrt{3}=d$. Then we have

$$
\int_{a}^{b} p(t, \eta) \Delta \eta<\int_{1}^{\sqrt{3}} \frac{\sqrt{1+\eta^{2}}+\eta}{4} \Delta \eta=\frac{1}{2}, \quad t \in \mathbb{T},
$$

and

$$
\int_{t_{0}}^{\infty} \int_{c}^{d} \omega(s, v) \Delta v \Delta s>\int_{1}^{\infty} \frac{-1}{8 s \sqrt{1+s^{2}}} \Delta s=\frac{-1}{8} .
$$

Therefore, by Theorem 3.3 with $\alpha=\frac{1}{2}$, (4.3) has a bounded nonoscillatory solution.

Example 4.4 Fixed $h>0$ and let $\mathbb{T}=h \mathbb{N}$. Consider the following equation:

$$
\left[x(t)+\int_{h}^{3 h} \frac{x(t+h \eta)}{t+3 \eta} \Delta \eta\right]^{\Delta}-\int_{h}^{3 h} \frac{h x\left(t+\frac{1}{h} v\right)}{t(t+h)(t+12 v)} \Delta v=0
$$

i.e., $p(t, \eta)=-\frac{1}{t+3 \eta}, \omega(t, v)=-\frac{h}{t(t+h)(t+12 v)}, a=h=c, b=3 h=d$. Then we have

$$
\int_{a}^{b} p(t, \eta) \Delta \eta>-\int_{h}^{3 h} \frac{1}{3 \eta} \Delta \eta=-\frac{1}{2}, \quad t \in \mathbb{T},
$$

and

$$
\int_{t_{0}}^{\infty} \int_{c}^{d} \omega(s, v) \Delta v \Delta s>\int_{h}^{\infty} \frac{h}{s(s+h)} \Delta s \int_{h}^{3 h} \frac{-1}{12 v} \Delta v=\frac{-1}{8}
$$

Therefore, by Theorem 3.4 with $\alpha=\frac{1}{2}$, (4.4) has a bounded nonoscillatory solution. 


\section{Conclusion}

In this paper, using Krasnoselskii's Fixed Point Theorem, we obtain some sufficient conditions for the existence of nonoscillatory solutions to the first-order neutral dynamic equation (1.1) in four cases. And we give some examples to illustrate the main results. A similar method can be used to prove the existence of nonoscillatory solutions for other dynamic equations.

\section{Funding}

This work is partially supported by National Natural Science Foundation of China (No. 11401116, No. 11761011, No. 11461003) and Natural Science Foundation of Guangxi (No. 2014GXNSFBA118003).

\section{Competing interests}

The authors declare that they have no competing interests.

\section{Authors' contributions}

All authors read and approved the final manuscript.

\section{Publisher's Note}

Springer Nature remains neutral with regard to jurisdictional claims in published maps and institutional affiliations.

Received: 20 September 2018 Accepted: 8 February 2019 Published online: 19 February 2019

\section{References}

1. Zafer, A.: On oscillation and nonoscillation of second-order dynamic equations. Appl. Math. Lett. 22, 136-141 (2009)

2. Gao, J., Wang, Q.: Existence of nonoscillatory solutions to second-order nonlinear neutral dynamic equations on time scales. Rocky Mt. J. Math. 43, 1521-1535 (2013)

3. Graef, J.R., Hill, M.: Nonoscillation of all solutions of a higher order nonlinear delay dynamic equation on time scales. J. Math. Anal. Appl. 423, 1693-1703 (2015)

4. Qiu, Y.: On nonoscillatory solutions tending to zero of third-order nonlinear dynamic equations on time scales. Adv. Differ. Equ. 2016, Article ID 10 (2016)

5. Mathsen, R., Wang, Q., Wu, H.: Oscillation for neutral dynamic functional equations on time scales. J. Differ. Equ. Appl. 10(7), 651-659 (2004)

6. Zhu, Z., Wang, Q.: Existence of nonoscillatory solutions to neutral dynamic equations on time scales. J. Math. Anal. Appl. 335, 751-762 (2007)

7. Gao, S., Chen, Z., Shi, W.: New oscillation criteria for third-order neutral differential equations with continuously distributed delay. Appl. Math. Lett. 77, 64-71 (2017)

8. Candan, T:: Oscillation criteria for second-order nonlinear neutral dynamic equations with distributed deviating arguments on time scales. Adv. Differ. Equ. 2013, Article ID 112 (2013)

9. Senel, M.T., Utku, N.: Oscillation criteria for third-order neutral dynamic equations with continuously distributed delay. Adv. Differ. Equ. 2014, Article ID 220 (2014)

10. Hassan, T.S., Grace, S.R.: Oscillation criteria for third order neutral nonlinear dynamic equations with distributed deviating arguments on time scales. Tatra Mt. Math. Publ. 61, 141-161 (2014)

11. Agarwal, R.P., Bohner, M., O'Regan, D., Peterson, A.: Dynamic equations on time scales: a survey. J. Comput. Appl. Math. 141, 1-26 (2002)

12. Bohner, M., Peterson, A.: Dynamic Equations on Time Scales: An Introduction with Applications. Birkhäuser, Boston (2001)

13. Chen, Y.: Existence of nonoscillatory solutions of $n$th order neutral delay differential equations. Funkc. Ekvacioj 35 , $557-570$ (1992)

\section{Submit your manuscript to a SpringerOpen ${ }^{\circ}$ journal and benefit from:}

- Convenient online submission

- Rigorous peer review

- Open access: articles freely available online

- High visibility within the field

- Retaining the copyright to your article

Submit your next manuscript at $\gg$ springeropen.com 